\title{
Intervista a Diego Brancaccio
}

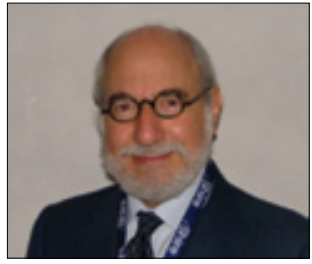

Prof. Diego Brancaccio

Università di Milano

diego.brancaccio@tiscali.it

Nel centro ove presti attività qual è la percentuale di pazienti monitorati per CKD-MBD stadio 3-5 e quali marker biologici usi a tal fine?

Come forse saprai, dopo aver lasciato la Direzione della UO di Nefrologia dell'Ospedale "San Paolo", sono ora il Direttore della UO di Emodialisi "Simone Martini” di Milano. Qui si pratica solo emodialisi (circa 60 pazienti) e abbiamo più recentemente avviato l'ambulatorio dell'uremia.

Dopo questa premessa, ora posso dire che tutti i pazienti dializzati sono monitorati (Ca, P, PTH almeno ogni 2 mesi, $25 \mathrm{OH}$ ogni sei mesi), mentre i soggetti con CKD hanno piani di controllo assai diversi tra di loro in relazione ai diversi livelli di PTH.

Nel centro ove presti attività pratichi il dosaggio della 25(OH)D e/o della 1,25(OH) D3? Se si, in quale tipo di paziente e in quale percentuale? Se no, perché ritieni di non doverla praticare?

Tutti i pazienti emodializzati vengono controllati su base regolare (primavera e autunno).

Per quanto attiene alla $1,25(\mathrm{OH})_{2} \mathrm{D} 3$ penso che ormai sia chiaro a tutti che questo dosaggio non ha senso se non per ricerca. Per meglio chiarire il problema vorrei riproporre due quesiti assurdi ma didattici, sulla scorta di quanto mi ha suggerito l'amico Slatopolsky da tempo: «Per quantificare la dose di insulina da somministrare per il controllo del profilo glicemico nei pazienti diabetici, doseremmo noi i livelli sierici della insulinemia o la glicemia? Analogamente, nei soggetti uremici: per il controllo dell'iperparatiroidismo secondario, doseremmo noi il calcitriolo sierico o il PTH?»
Applichi la correzione di $25(\mathrm{OH}) D$ in caso di carenza? Perché, con quale protocollo e in quale tipo di paziente?

Esistono ampi studi che indicano come ridotti livelli di 25OHD3 si associno ad outcomes sfavorevoli sia in termini di morbilità che di mortalità generale e cardiovascolare. Tuttavia non esiste alcuno studio prospettico che dimostri che la supplementazione di 25OHD3 sia vantaggiosa per i pazienti uremici. In ogni caso, personalmente ritengo utile la somministrazione di 25OHD3 per valori sierici inferiori a $15 \mathrm{ng} \mathrm{mL} \mathrm{e,} \mathrm{per} \mathrm{tranquilliz-}$ zare l'animo dei meno esperti, vorrei segnalare che è assai raro osservare accumulo di 25OHD3 nell'organismo con conseguente ipercalcemia nei soggetti uremici trattati con questo ormone ed è stato da più autori suggerito che anche livelli sierici di 100-120 ng mL non si associano a ipercalcemia.

Nel centro ove presti attività in quale percentuale o in quali casi clinici particolari pratichi la biopsia ossea?

No.

Nel centro ove presti attività in quale percentuale $e$ in quali stadi di CKD usi indagini strumentali per la ricerca delle calcificazioni vascolari? Quali indagini utilizzi?

Antonio Bellasi, che ha lavorato a lungo con me e successivamente tre anni da Paolo Raggi, ha pubblicato quattro anni fa un buon lavoro su Kidney International in cui dimostra che la misura del calcium score coronario mediante EBCT, nei soggetti uremici, si correla con la deposizione di calcio aortico misurato con una semplice radiografia dell'addome in L-L. Dunque ritengo personalmente che la misura del Coronary Calcium Score mediante EBCT o TAC sia ormai da considerare solamente per protocolli scientifici, ma non più per il semplice follow-up clinico.

Non abbiamo accesso, almeno per il momento nel nostro centro, alla misura della pulse wave velocità, anch'essa di grande significato clinico-funzionale. Essa è al mo- 
mento attuale ancora poco usata in Italia.

Nel centro ove presti attività qual è la percentuale di pazienti in trattamento sostitutivo che resta nel range di normalità (secondo le linee guida SIN) per Ca, P e PTH,?

Questa domanda è mal posta poiché, almeno nei pazienti uremici naive, la quota di pazienti dializzati che è all'interno dei range DOKI non supera la soglia del 10\% qualora si consideri la tripletta di tutti e tre i parametri. Quando i pazienti vengono trattati con Paracalcitolo o Calciomimetico questa percentuale sale fino a $30-45 \%$. Tuttavia la mia obiezione si riferisce al fatto che riportare i pazienti entro i limiti delle Linee Guida non significa molto. Spesso di tratta di una cosmesi dei parametri e a questo proposito vorrei citare un bel titolo di un breve lavoro apparso recentemente che mette in luce proprio questo: "treat the patient not the lab values, NDT vol 3, 81-83, 2010".

Nel centro ove presti attività qual è la percentuale dei vari chelanti usati per ricercare il controllo del Ca-P nei pazienti in trattamento sostituitvo (anche in off-label treatment)?

Anche questa domanda mi mette a disagio, tuttavia vorrei segnalare che tutti i miei pazienti emodializzati assumono una quota pur ridotta di chelanti a base di calcio (max: $2000 \mathrm{mg}$ die). Detto questo, circa il 30\% assume anche Renagel e un altro 30\% Lantanio Carbonato.

Nel centro ove presti attività qual è la percentuale di pazienti in CKD 3-5 sottoposta a dieta ipoproteica per il controllo della iperfosforemia?

Ritengo che debba essere applicata con una semplice riduzione di pochi alimenti (vedi successive risposte).

Nel centro ove presti attività quale percentuale di pazienti in trattamento sostitutivo che assumono vit-D o analoghi, calciomimetico, o entrambi?

Oltre il 60\% dei pazienti ricevono 25OHD3 su base regolare. Inoltre un $30-40 \%$ riceve anche Paracalcitolo e un $10 \%$ Cinacalcet.

Vorrei segnalare ora un elemento importante ai fini di una corretta strategia per il controllo dell'assetto Ca-P: la supplementazione di 25OHD3 non ha niente a che vedere con il trattamento delliperparatiroidismo secondario dei pazienti uremici. Francamente credo che la carenza di 25OHD3 sia espressione di una carenza nutrizionale la cui supplementazione oggi ha carattere prudenziale. Credo invece sia maturo il tempo per considerare essenziale l'attivazione dei VDR in modo più ampio, con agonisti selettivi che, accanto al controllo ottimale della secrezione di PTH, possano portare a una migliore cardioprotezione, come più volte dimostrata in numerosi vastissimi studi clinici - sia pur di associazione e a carattere retrospettivo - supportati anche da recenti studi sperimentali.

Tra gli studi osservazionali, ma a carattere prospettico, vorrei citare lo studio FARO (ora in corso di pubblicazione sul Journal of Nephrology); esso è una survey italiana che ha incluso oltre 2300 pazienti soggetti emodializzati (appartenenti a 28 Centri Dialisi), seguiti nel corso di 18 mesi, e in cui è stato valutato oltre che il controllo del profilo del PTH anche la mortalità generale e cardiovascolare. Tra vari sottogruppi considerati in questo studio non randomizzato, è emerso che il gruppo di pazienti trattati con Paracalcitolo avevano un migliore attesa di vita sia in termini di mortalità generale che cardiovascolare rispetto a coloro che erano trattati con Calcitriolo. Inoltre l'aggiunta di Calciomimetico non aggiungeva vantaggi in termini di outcome qualora aggiunto alla terapia con Paracalcitolo.

Questi elementi, accanto ai dati emersi nella letteratura dell'ultima decade, fanno capire come l'attivazione dei VDR sia essenziale nei soggetti uremici e come l'impiego degli agonisti selettivi possa rappresentare uno strumento terapeutico che va ben al di là del semplice controllo del PTH.

L'altra grande evoluzione nel trattamento dell'iperparatiroidismo secondario è l'impiego dei calciomimetici, e più recentemente Block (Kidney International, agosto 2010) ha riportato come in un'ampia casistica, pur retrospettiva, di soggetti dializzati, coloro che sono stati trattati con Cinacalcet avevano una miglior attesa di vita rispetto al gruppo non trattato con Ca mimetici.

Dopo oltre un trentennio di osservazioni, esperienze, studi e ricerche, la fisiopatologia dell'iperparatiroidismo secondario non è ancora del tutto chiarita. Calcio, fosforo, recettori della vit-D o recettori sensibili del calcio, ormoni fosfatotropi e forse l'esistenza di un recettore cellulare del fosforo, sono tutti "in carriera" assieme al primo attore il PTH (almeno nella cronologia storica): si è in grado di prevedere un ordine e chiarimento delle cose o dobbiamo ancora e solo predire?

Domanda importante e ben posta: francamente penso che la chiave della storia sia nell'espansione del pool del Fosfato. Ciò porta a una cascata di eventi negativi ormai ben noti (anche se ogni anno sul palcoscenico si muovo- 
no nuovi attori) tra cui primeggia la ridotta attività della 1-alfa-idrossilasi renale, con conseguente riduzione del calcitriolo sierico, già ridotto per effetto dell'FGF23. Il tutto porta anche a uno spostamento verso destra della curva sigmoidale $\mathrm{Ca} / \mathrm{PTH}$ per cui le paratiroidi divengono più sorde allo stimolo calcemico. Spostare nuovamente la curva $\mathrm{Ca} / \mathrm{PTH}$ verso sinistra è possibile: per questo scopo bisogna ridurre il $\mathrm{P}$ nella dieta, dare Attivatori dei VDR e infine anche i Calciomimetici qualora necessari. Questa domanda tuttavia meriterebbe una risposta lunga, che qui non troverebbe spazio, ma vorrei segnalare qui il ruolo centrale dell'accumulo del $\mathrm{P}$ nell'organismo del soggetto uremico che può avvenire pur con livelli sierici di fosfatemia controllati. Ne emerge il ruolo della dieta (pur a livelli non eroici) già nelle prime fasi della CKD.

Fosforo chiama "ormone"! Rispondono PTH, FGF23 e Klotho mentre $1,25(\mathrm{OH})_{2}$ D3 resta a guardare. Una relazione pericolosa? Più di un triangolo (fisiopatologico)? Un paradigma o cosa?

Dati assai suggestivi indicano che l'aumento della fosforemia nella popolazione con funzione renale normale si associa a un aumento del rischio cardiovascolare in termini di morbilità e mortalità. L'organismo si difende e, per mantenere l'omeostasi del P, attiva due ormoni, entrambi con azione fosfaturica: il PTH e l'FGF23 con attività metaboliche diverse ed entrambi attivati nella gran parte dei pazienti renali già dalle prime fasi della IRC.

Ma qui sta il punto: esiste una serie di studi ben fatti che indicano come anche modesti livelli di PTH si associno a un aumento della mortalità nei soggetti uremici, così come anche per aumentati livelli di FGF23. Dunque, se vogliamo essere concreti, sia la nostra dieta che la dieta del paziente renale dovrà essere meno ricca in fosfato: dovremmo ridurre l'alimentazione carnea e contenere i formaggi anche nella normale vita quotidiana ai nostri pazienti ma anche a noi stessi?
Adynamic bone disease: una (relativamente) nuova entità classificativa o una patologia emergente? Sovrastimata o snobbata?

Penso che almeno un terzo dei pazienti uremici abbia un osso a basso turnover le cui ripercussioni sul piano scheletrico sono significative ma non particolarmente allarmanti (lieve aumento della fragilità ossea e ridotta capacità di produrre il callo osseo). Ciò che invece preme sottolineare è che in queste condizioni si perde la preziosa funzione di buffer che l'osso fisiologicamente possiede e in questo modo ogni sovraccarico (acuto o cronico) di Calcio e/o Fosfato si traduce in un travaso di ioni divalenti nei tessuti molli e specificatamente nell'albero arterioso anziché nel tessuto osseo.

La diagnosi si basa sull'osservazione seriale dei livelli di PTH e calcemia il cui profilo va modulato sospendendo farmaci o riducendo il Calcio nel dialisato e idealmente attraverso la biopsia ossea.

Nonostante tutto (linee guida comprese, SIN, K/DOKI, KDIGO) nella maggioranza dei casi i target terapeutici per calcio, fosforo e PTH non vengono centrati e soprattutto mantenuti. Quale commento ti stimola tale insufficiente raggiungimento?

Perché dunque è così difficile raggiungere i target DOKI o K-DIGO? Personalmente ritengo che essi non debbano essere considerati rigidamente. Se vogliamo essere crudi, vorrei segnalare come la maggior parte di pazienti trattati aggressivamente per ridurre il PTH al di sotto di $300 \mathrm{pg} / \mathrm{mL}$, oggi secondo le linee K.DIGO non sarebbero da trattare. Inoltre, la lettura delle nuove linee K.DIGO fa capire come ogni singolo parametro, di per sé, sia poco indicativo e che è l'analisi di tutto il profilo globale che può essere di guida per il nefrologo clinico. In ogni caso ritengo valida la mia citazione in cui si suggerisce di seguire e trattare il paziente e non solo i numeri di laboratorio. 